\title{
Health-related quality-of-life among patients with premature ovarian insufficiency: a systematic review and meta-analysis
}

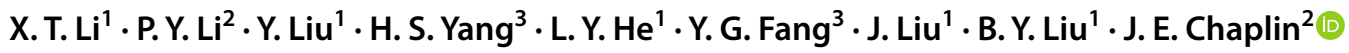

Accepted: 1 October 2019 / Published online: 16 October 2019

(c) The Author(s) 2019

\begin{abstract}
Purpose To systematically review studies investigating health-related quality-of-life (HrQoL) in patients with premature ovarian insufficiency (POI), to examine questionnaires used and to conduct a meta-analysis of control studies with normal ovarian function.

Methods Data sources: PubMed, Embase, Web of science, CNKI, and CQVIP, searched from inception until June 2018. The search strategy was a combination of medical (e.g. POI), subjective (e.g. well-being) and methodological (e.g. questionnaires) keywords. PRISMA guidelines were used to assess outcome data quality/validity by one reviewer, verified by a second reviewer. Risk of bias within studies was evaluated. A meta-analysis compared HrQoL in patients and non-patients. Due to measurement differences in the studies, the effect size was calculated as standard mean difference.

Results We identified $6869 \mathrm{HrQoL}$ studies. Nineteen geographically diverse studies met inclusion criteria, dated from 2006, using 23 questionnaires. The meta-analysis included six studies with 645 POI participants (age 33.3 \pm 5.47 ) and 492 normal-ovarian control subjects (age $32.87 \pm 5.61$ ). Medium effect sizes were found for lower overall HrQoL (pooled $\mathrm{SMD}=-0.73,95 \% \mathrm{CI}-0.94,-0.51 ; I^{2}=54 \%$ ) and physical function (pooled $\mathrm{SMD}=-0.54,95 \% \mathrm{CI}-0.69,-0.39$; $\left.I^{2}=55 \%\right)$. Heterogeneity was investigated. Effect sizes varied for sexual function depending on the measure $(\mathrm{SMD}=-0.27$ to -0.74 ), overall HrQoL (SF-36) had the largest effect size $(-0.93)$ in one study. The effect sizes for psychological and social HrQoL were small.

Conclusion POI is associated with low-to-medium effect size on HrQoL compared to normal ovarian controls. The greatest effects are found in general HrQoL and most sexual function areas. Condition-specific questionnaires and RCTs are recommended for further investigation.
\end{abstract}

Keywords Surveys and questionnaires · Menstruation disturbance · Gynaecology $\cdot$ Women's health

\begin{tabular}{|c|c|c|}
\hline & Abbreviations & \\
\hline & CAMS-R & $\begin{array}{l}\text { The Cognitive and Affective Mindful- } \\
\text { ness Scale V Revised }\end{array}$ \\
\hline & CES-D & Epidemiologic Studies Depression \\
\hline ectronic supplementary material The online version of this & & Scale \\
\hline $\begin{array}{l}\text { ticte supplementary material ine online version of this } \\
\text { ticle (https://doi.org/10.1007/s11136-019-02326-2) contains }\end{array}$ & DHEA & Dehydroepiandrosterone \\
\hline upplementary material, which is available to authorized users. & DISF-SR & Derogatis Interview for Sexual Func- \\
\hline B. Y. Liu & DOR & Diminished ovarian reserve \\
\hline baoyanjournal@163.com & DSM-IV (SCID) & Diagnostic and Statistical Manual of \\
\hline Institute of Basic Research in Clinical Medicine, China & & Mental Disorders (fourth edition) \\
\hline Academy of Chinese Medical Sciences, Beijing, China & FACIT-Sp-12 & Functional Assessment of Chronic Ill- \\
\hline Department of Pediatrics, The Queen Silvia Children's & & ness Therapy-Spiritual Well-Being \\
\hline Hospital, Institute of Clinical Sciences, Sahlgrenska & & Scale \\
\hline $\begin{array}{l}\text { Academy, University of Gothenburg, } 41685 \text { Gothenburg, } \\
\text { Sweden }\end{array}$ & FACIT-Sp-Ex & $\begin{array}{l}\text { Functional Assessment of Chronic Ill- } \\
\text { ness Therapy-Spiritual Well-Being }\end{array}$ \\
\hline $\begin{array}{l}\text { Institute of Acupuncture and Moxibustion, China Academy } \\
\text { of Chinese Medical Sciences, Beijing, China }\end{array}$ & & Scale Expanded \\
\hline
\end{tabular}




$\begin{array}{ll}\text { FANLTC } & \text { Functional Assessment of Non-Life- } \\ \text { FertiQoL } & \begin{array}{l}\text { Threatening Conditions } \\ \text { International Fertility Quality of Life } \\ \text { Questionnaire }\end{array} \\ \text { FSFI } & \text { Female Sexual Function Index } \\ \text { FSH } & \text { Follicle-Stimulating Hormone } \\ \text { GCS } & \text { Greene Climacteric Scale } \\ \text { HrQoL } & \text { Health-related quality of life } \\ \text { HRT } & \text { Hormone replacement therapy } \\ \text { IHD } & \text { Ischaemic heart disease } \\ \text { LEU } & \text { Life events scale } \\ \text { NOS } & \text { Newcastle-Ottawa Scale } \\ \text { PANAS } & \text { Positive and Negative Affect Schedule } \\ \text { PCOS } & \text { Polycystic ovarian syndrome } \\ \text { PM } & \text { The Pearlin Mastery Scale } \\ \text { POF } & \text { Premature ovarian failure } \\ \text { POI } & \text { Premature ovarian insufficiency } \\ \text { POR } & \text { Poor ovarian responders } \\ \text { PRQ85 } & \text { Personal Resource Questionnaire 1985 } \\ \text { QOL } & \text { Quality of life } \\ \text { SF-36 } & \text { The 36-Item Short Form Survey from } \\ & \text { the RAND Medical Outcomes Study } \\ \text { SMD } & \text { Standard Mean Difference } \\ \text { SPEQ } & \text { Sexual Personal Experiences } \\ & \text { Questionnaire } \\ \text { STAI } & \text { State-Trait Anxiety Inventory } \\ \text { TABP/TCBP } & \text { Type A/C behavior pattern } \\ \text { TCM } & \text { Traditional Chinese Medicine } \\ \text { WHOQoL-BREF } & \text { World Health Organization Quality of } \\ & \text { Life } \\ \text { YMA } & \text { Young Menopause Assessment } \\ & \end{array}$

\section{Introduction}

Thanks to medical advances, the living condition of women with premature ovarian insufficiency (POI) has gained more attention in recent years [1]. POI is a clinical syndrome defined by loss of ovarian activity before the age of 40, associated with menstrual disturbance, raised gonadotropins and low estradiol [2]. Although proper diagnostic accuracy in POI is lacking, the European Society of Human Reproduction and Embryology (ESHRE) has developed guidelines on management of women with premature ovarian insufficiency [2] in which they recommend the following diagnostic criteria for POI: (i) oligo/ amenorrhea for at least 4 months, and (ii) an elevated FSH level $>25$ IU/l on two occasions $>4$ weeks apart. The nomenclature has changed over the years and POI has been referred to as premature ovarian failure, premature menopause, and premature ovarian dysfunction [3]. Earlier studies often used the term premature ovarian failure (POF) and more recent articles have used POI. It should also be noted that in POI serum follicle-stimulating hormone (FSH) levels are often found to exceed the diagnostic definition in studies of POI and are noted in several studies to be above 40 IU/L [2-4]. An earlier study reported the prevalence of POI in women under 30 years old estimated to be $0.1 \%$, while the incidence of menopause in women before the age of 40 is approximately $1 \%$ [5]. In recent years, studies have investigated the prevalence of patients with POI in different countries. For example, one article reported a higher prevalence (1.9\%; 95\% CI 1.7-2.1) of POI in women before the age of 40 in Sweden [6] and another article reported $0.91 \%$ (95\% CI $0.81-1.02 \%$ ) in Estonia [7]. There has been a long-standing confusion over the various terms such as poor ovarian responders (POR), premature menopause and diminished ovarian reserve (DOR) $[2,3,8,9]$. It is important to distinguish these conditions from POI because women with POI face more challenges than diminished fertility, and have different management needs $[2,10]$. Only $5-10 \%$ of women with POI may be able to spontaneously conceive and deliver a child [11]. In addition, women with POI suffer from amenorrhea-related symptoms [12] psychological problems [13, 14], increased risk to cardiovascular health $[15,16]$ and to bone health [17]. POI is a condition that is influenced by genitourinary and sexual function [18] and neurological dysfunction [19] in both the short- and long-term and can lead to premature death [20]. The best option to relieve symptoms and protect POI patients against serious morbidity related to prolonged estrogen deficiency is hormone replacement therapy (HRT). However, HRT is just a mimic of normal physiological endocrinology, which has no evidence to improve the ovary function [2]. Consequently, patients with POI are at risk of poor health quality despite available treatment options. Quality of life (QoL) is a broad multidimensional concept that usually includes subjective evaluations of both positive and negative aspects of life [21]. While, health-related quality of life (HrQoL) focus on the effects of a disease on an individual's health and its treatment [22-25] encompassing physical, psychological, and social functioning $[23,26]$ and presents an avenue for the evaluation of the consequences of experiencing premature ovarian insufficiency. This review aimed to investigate studies of women with POI, which have included measures of HrQoL, in order to evaluate effect sizes and in addition to identify the measurement instruments used. A meta-analysis was conducted of the studies that reached quality standards and which compared the HrQoL outcomes among patients with POI with a control group consisting of normal ovary function women. 


\section{Materials and methods}

This study followed the Preferred Reporting Items for Systematic Reviews and Meta-analyses (PRISMA) [27] reporting guideline (Online Resource ESM_1). A submission to the ethics committee of the Clinical Basic Medicine Institute, China Academy of Chinese Medical Sciences was sought. The Ethics committee judged that ethical approval was not required for this research (ref 2019/1).

\section{Search strategy and data selection}

An electronic search of the six databases was undertaken from database inception to June 2018. PubMed/MEDLINE and 'Web of science' provided a broad coverage of the biomedical literature, including reproductive biology and clinical medicine. EMBASE was included because it has greater coverage of European and non-English language publications and topics such as alternative medicine. China National Knowledge Infrastructure (CNKI), WanFang database and Chongqing VIP information (CQVIP) were included to ensure that no Asian publications were missed. Searches were conducted without restrictions with respect to publication year, language, type or setting of study or accessibility to full-text articles. A combination of keywords and database specific terms was used (premature ovarian insufficiency OR premature ovarian failure OR diminished ovarian reserve OR poor ovarian response OR premature menopause OR hyper-gonadotropic hypogonadism OR elevated gonadotrophins OR triad of amenorrhea OR estrogen deficiency) AND (well-being OR health outcome OR quality-of-life OR health-related quality of life) AND (questionnaire OR instrument OR patient reported outcome). Strategies differed in the different databases depending upon the information structures. The details of the different search strategies are provided in the online resource materials (online resource ESM_2). The process of article selection is outlined in Fig. 1 with a description of predefined criteria for selection. One author (XT Li) was mainly responsible for screening the titles and abstracts. Articles identified were independently read and discussed with two more authors (HS Yang, PY Li) to ensure an unbiased selection. Some studies of post-menopause have used instruments such as the MSQOL [28, 29] however this is not a measure of subjective quality-of-life and was therefore not included in this review. No additional articles were identified through the manual search. Studies describing the construction and validity of the HrQoL questionnaires used in the studies were also evaluated. If information on construction and validity was sparse, contact was attempted with the author responsible for the development of the questionnaire.

\section{Criteria to select articles}

The inclusion criteria for empirical investigation studies of adults with POI was that HrQoL was a primary or secondary outcome. Studies with participants from hospitals and long-term care facilities or with specific conditions (e.g. Turner syndrome or anorexia) or where abstracts only were found were included in the literature in order to be able to extract data on the questionnaires used but excluded from the meta-analysis. No restrictions were placed on the geographic, soioeconoimic or ethinic backgrounds of any of the participants. There was no restriction in terms of treatment, both randomized and non-randomized trials were included. Exclusion criteria for the systematic review were duplicate publications or reviews, studies that did not include outcomes from a HrQoL questionnaire. Exclusion criteria for the meta-analysis were articles which lacked relevant data for investigation and studies without a normal ovary function control group.

\section{Critical appraisal: assessment of bias in the studies}

The quality of eligible articles was assessed at the study level using the Newcastle-Ottawa Scale (NOS) for nonrandomized cohort studies [30]. Each article was awarded a 'star' or score out of four for selection bias, two for comparability and three for bias in the outcome assessment, with a maximum total score of nine points. The NOS score was used to assess differences in study quality scores $>6$ high; $4-6$ medium, $<4$ low [31]. The scoring system and evaluation is provided in the Online Resource ESM_3. Two authors (XT Li, PY Li) independently evaluated the findings of each study to ensure an unbiased assessment.

\section{Meta-analysis}

A meta-analysis investigated the outcome of HrQoL in patients with POI compared with a normal ovary function reference population. Review Manager (Version 5.3. Copenhagen: The Nordic Cochrane Centre, The Cochrane Collaboration, 2014) was used. The estimated value and 95\% confidence interval (95\% CI) of the effect size was calculated by Standard Mean Difference (SMD) [32]. The SMD is used as a summary statistic in meta-analysis when the studies all assess the same outcome but measure it in a variety of ways [33]. Cohen [34] suggested that $d=0.2$ be considered a 'small' effect size, 0.5 represents a 'medium' effect size and 0.8 a 'large' effect size. The size of heterogeneity among studies after combination was determined via $I^{2}$ statistic: $0 \%$ to $40 \%$ : might not be important; $30 \%$ to 
Fig. 1 The article selection process and criteria for selection for the literature review and meta-analysis

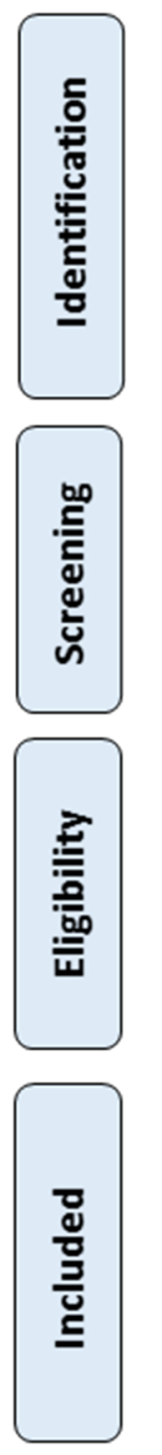

60\%: may represent moderate heterogeneity; $50 \%$ to $90 \%$ : may represent substantial heterogeneity; $75 \%$ to $100 \%$ : considerable heterogeneity [35]. If there was no heterogeneity among studies, a fixed effects model was applied

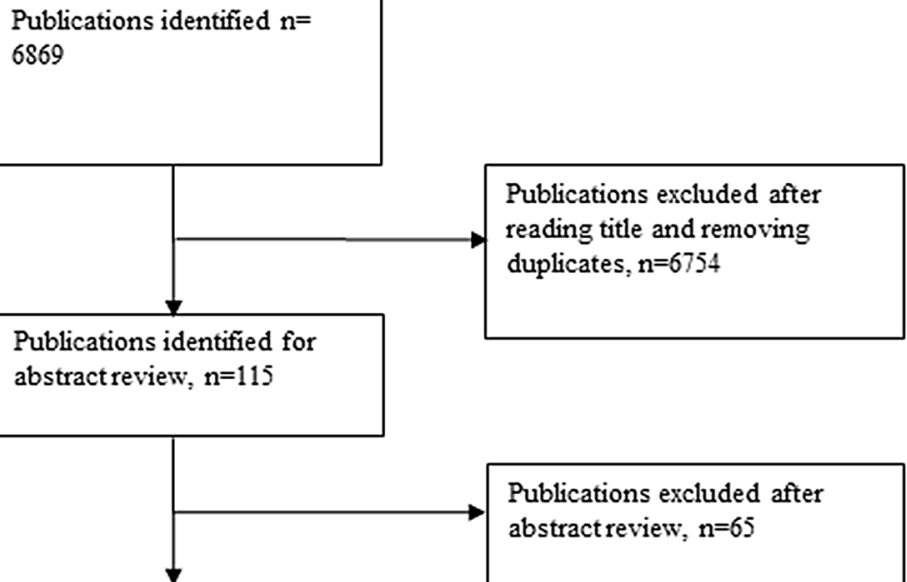

Publications identified for article review, $n=50$

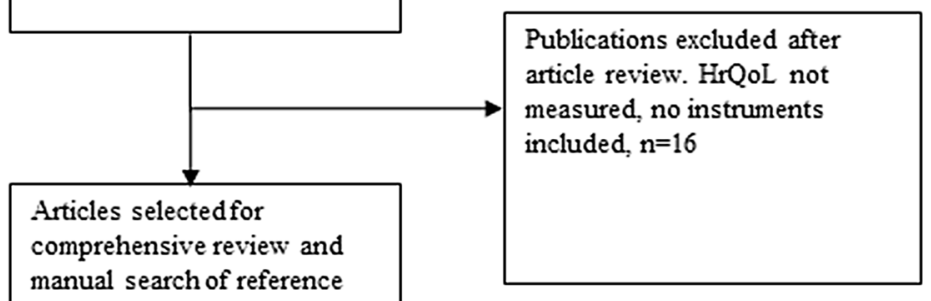
list, $n=34$

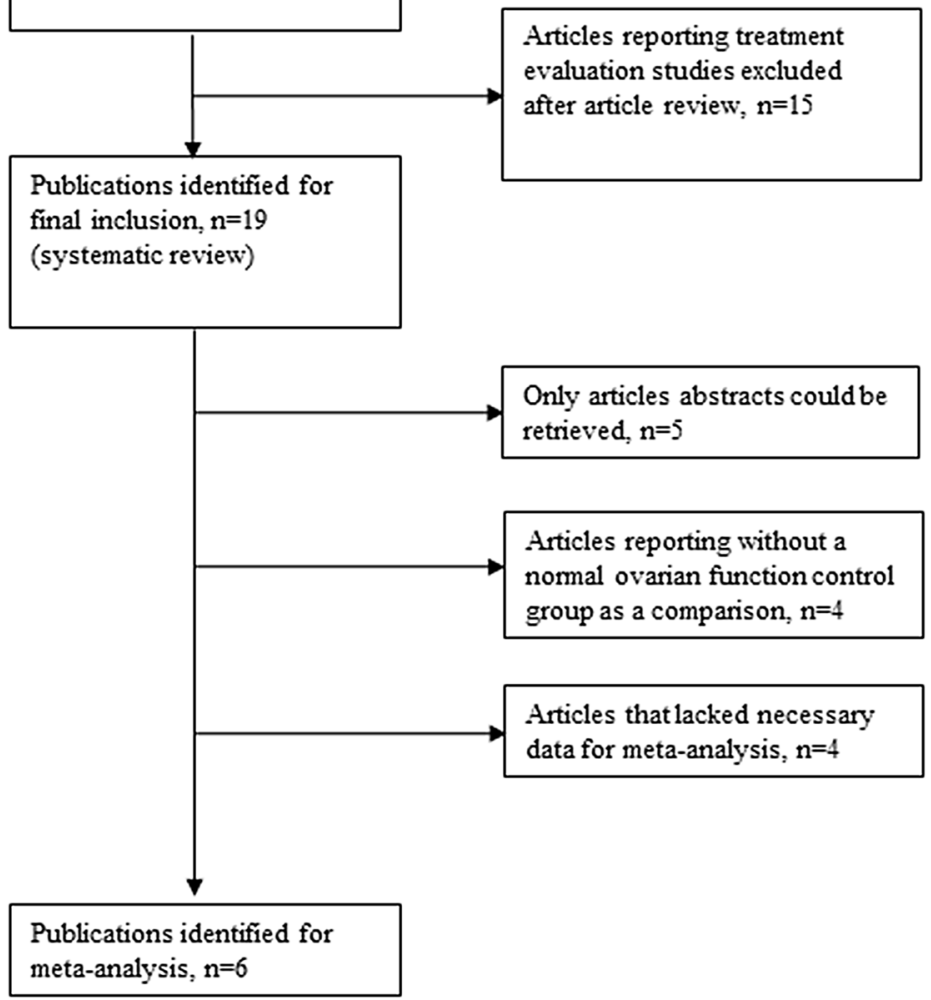

for meta-analysis; if there was statistical heterogeneity, the sources of heterogeneity were further analyzed, and a random effects model was adopted for meta-analysis. According to the same questionnaires used and same 
specific domain evaluated, the effect sizes were divided into subgroups. This systematic review and meta-analysis were performed and reported according to the PRISMA guidelines. The PRISMA checklist is included as Online Resource_3.

\section{Results}

Thirty-four studies matched the inclusion criteria and were included for review. Fifteen articles were related to treatment evaluation while 19 articles examined elements of HrQoL (Tables 1,2). In five of these studies only the abstracts were available for examination [36-40]. These articles were all published between 2006 and 2018. Eighteen articles were cross-sectional studies [36-53] two of which included case-controls $[43,51]$. One article reported only case-control data [54]. Nine articles described HrQoL among patients with the nomenclature of POI [36, 39, 40, 42, 47, 49, 51-53] and ten articles described HrQoL among patients with the previous nomenclature of POF [37, 38, 41, 43-46, 48, 50, 54]. Thirteen articles had control groups [39-46, 48, 49, 51, $53,54]$ and nine of these had a control group of women with normal ovarian function [41-46, 51, 53, 54], six of these had sufficient information to be included in the meta-analysis $[41-45,54]$. None of the studies used proxy-reports from family members as part of the evaluation. Reported studies had varying sample sizes; the largest sample size was 340 women [46]. The studies were geographically diverse including China [41, 44-46], UK [37, 38, 50], America [36, 39, 40, 42, 49, 51-53], Brazil [43, 54], Australia [48] and multi-national studies [47] (Fig. 1 and Tables 1,2).

\section{Domains of HrQoL examined}

The definition of HrQoL used in the studies is derived from the domains of the questionnaires used to measure HrQoL. Among the 19 articles examining HrQoL, seven studies included a measure of overall $\mathrm{HrQoL}$ as measured by either a generic questionnaire (SF-36, WHOQoL-BREF) [37, 43, 44, $50,54]$ or measured in relation to fertility or sexual function $[42,45,50,54]$. Nine studies focused on psychiatric aspects including depression and meaning in life [36, 38-40, 49-53]. Four articles used the POI related symptom questionnaires $[38,47,48]$ Only one of these [50] used a condition specific instrument designed for POI (Young Menopause Assessment (YMA) [50]). One study evaluated the aspect of social function: perceived social support [53]. The reduced HrQoL among patients with POI was mentioned in all 19 articles. A summary of the studies is found in Tables 1,2 .

\section{Overall HrQoL}

Three articles described factors correlated with lower HrQoL in POI populations: one article reported that orgasm and sexual satisfaction were correlated with all QOL domains [54]; a second article analysed character traits of POI patients [45], which showed that older patients, with primary infertility and who had had children had lower HrQoL scores than patients who were of younger age, secondary infertility or had previously given birth. In one article [44] different Traditional Chinese Medicine (TCM) syndromes were considered as summaries of symptoms of the pathogenesis of disease development [55]. These syndromes included insufficiencies of liver and kidney or asthenia of both the spleen and kidney. It was noted that patients with deficiency of liver and kidney had the lowest overall QOL scores (Table 3).

\section{Physical function and symptoms}

Physical health of the women with POI was consistently reported to be significantly lower than controls. A number of physical function symptoms were explored including experience of physical pain [43] sexual function [42, 54] arousal, lubrication, orgasm and satisfaction, and sexual behaviour/experiences $[42,50,54]$. In addition, menopause symptoms such as vasomotor symptoms, mood swings and mental fog, hair loss, dry eyes, cold intolerance, joint clicking, tingling in limbs and low blood pressure were found at a high rate in patients with POI [47].

\section{Psychological function and psychosocial aspects}

Women with spontaneous POI were reported to score adversely on all measures of psychological functioning [43, 51] with higher negative feelings such as "blue mood" [56], despair, anxiety, and depression or had a negative impact on their self-image and confidence [50]. This population also had a high rate of mental health medication use and counselling [51] and a risk for depression [49]. Some articles analysed the factors related to these negative feelings. Adverse affective symptoms were associated with a lower perceived level of control [39]. One article reported illness uncertainty and lack of purpose in life as a significant independent factor associated with anxiety [51]. Scores on the Spiritual WellBeing scale were also associate with POI and were found to reduce with increased age [52]. 







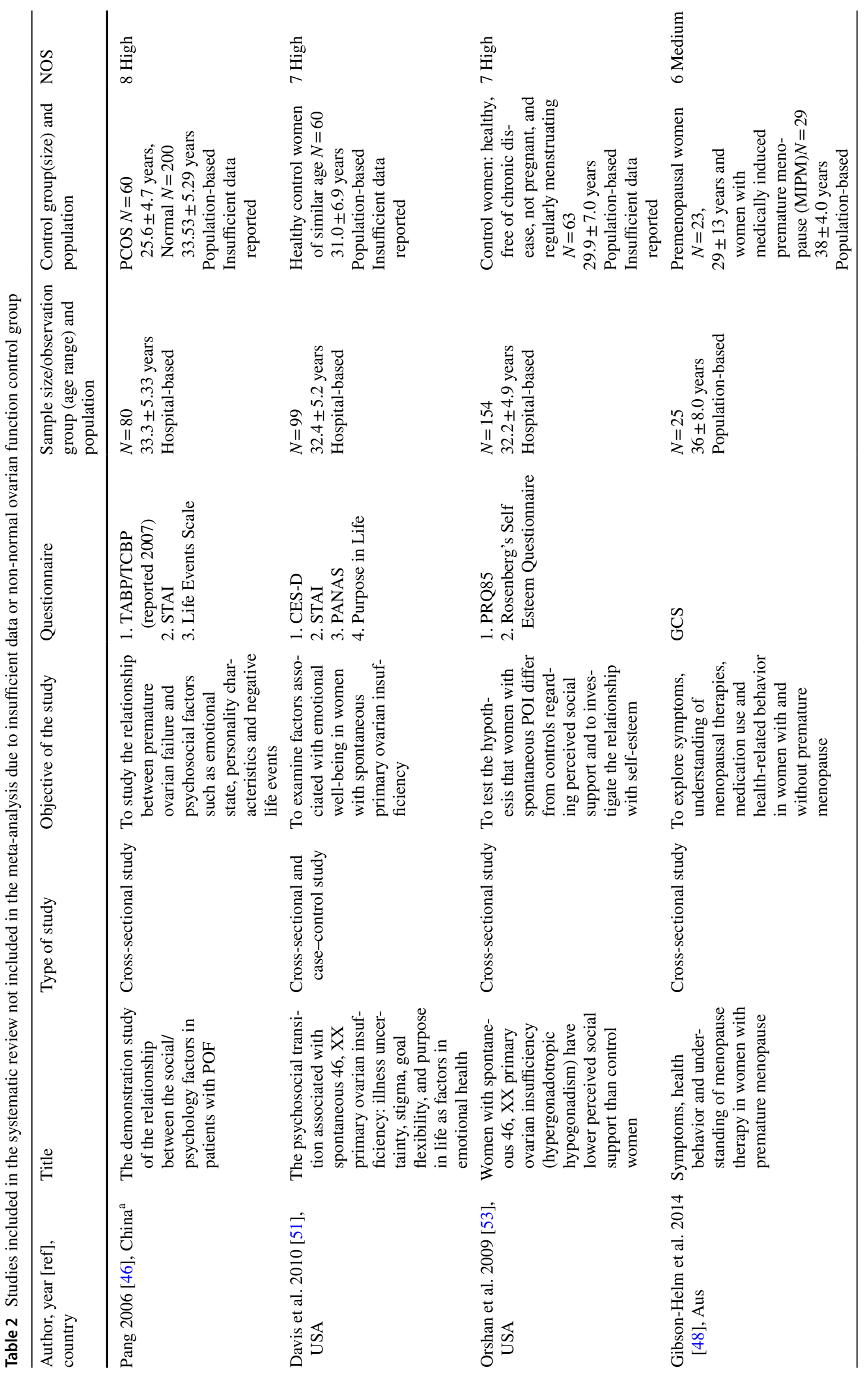




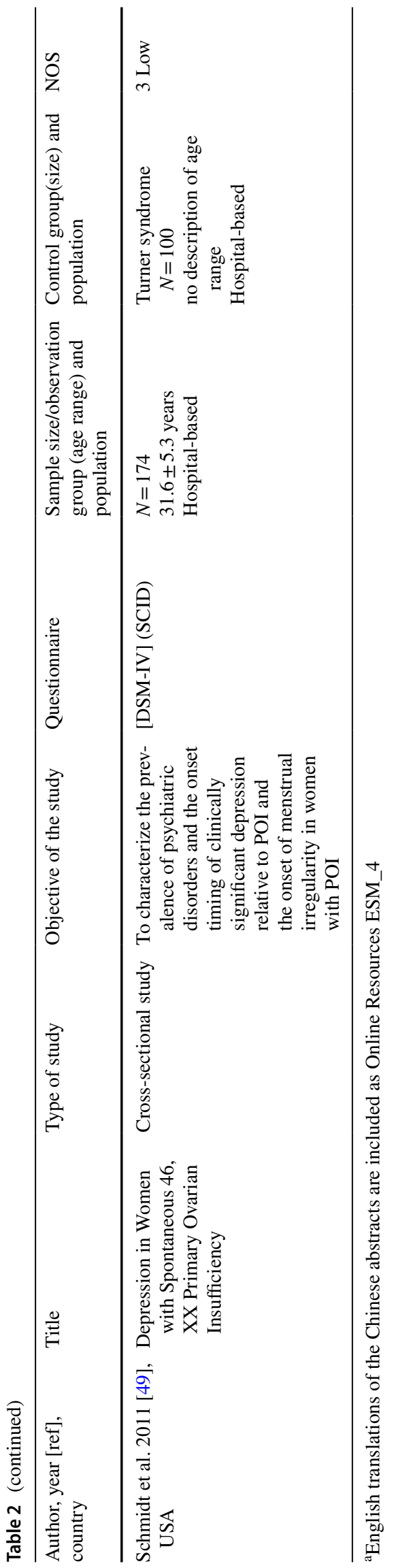

\section{Social function}

Marital relationship and social support were reported to be significantly lower in POI patients [45]. Social relationships were found to have a negative influence of sexual function such as arousal, orgasm, satisfaction and pain [53, 54]. However, other articles reported no significant differences found with respect to the social relationships or support $[43,46]$.

\section{Questionnaires}

In total, twenty-three different questionnaires had been used in the nineteen articles identified for review (Table 4). The most frequently used questionnaires were the two generic HrQoL: World Health Organization Quality of Life (WHOQoL-BREF) [62-64], and the 36-Item Short Form Survey from the RAND Medical Outcomes Study (SF-36) [65-67] which were used in five studies. Between 1 and 4 questionnaires were used in each study, $50 \%$ of the studies only used one questionnaire. Those studies that used four concentrated on the psychological aspects of the condition and were mainly from the same research group at NIH in the US and reported in Abstract form. Other studies combined generic questionnaires with condition specific issues e.g. sexual or menopause specific questionnaires. Only one study [50] used a POI specific questionnaire (Young Menopause Assessment (YMA) [Unpublished]. This was used in combination with a sexual function questionnaire (Sexual Personal Experiences Questionnaire (SPEQ) [73]) a psychological questionnaire (Rosenberg's Self Esteem Questionnaire [74-77]) and a generic questionnaire (SF-36 Short Form Survey from the RAND Medical Outcomes Study (SF-36) [65-67]). All the HrQoL instruments used are described in Table 4, a more detailed summary of the six questionnaires used in the studies included in the meta-analysis can be found as Online Resource ESM_5.

\section{Synthesis of results and risk of bias (results of meta-analysis)}

Six studies were included in the meta-analysis [41-45, 54] (Fig. 2) with 645 POI participants and 492 normal-ovarian controls. Where data on average age was available the POI group had a pooled mean age of $33.3 \pm 5.47$; and the control group a pooled mean age of $32.87 \pm 5.61$.

At the overall HrQoL level (Fig. 2a) four studies [42, 44, $45,54]$ had lower level of HrQoL recorded in the POI group (pooled SMD $=-0.73,95 \% \mathrm{CI}-0.94,-0.51 ; I^{2}=54 \%$ ) as compared to a normal ovarian control group. The pooled 


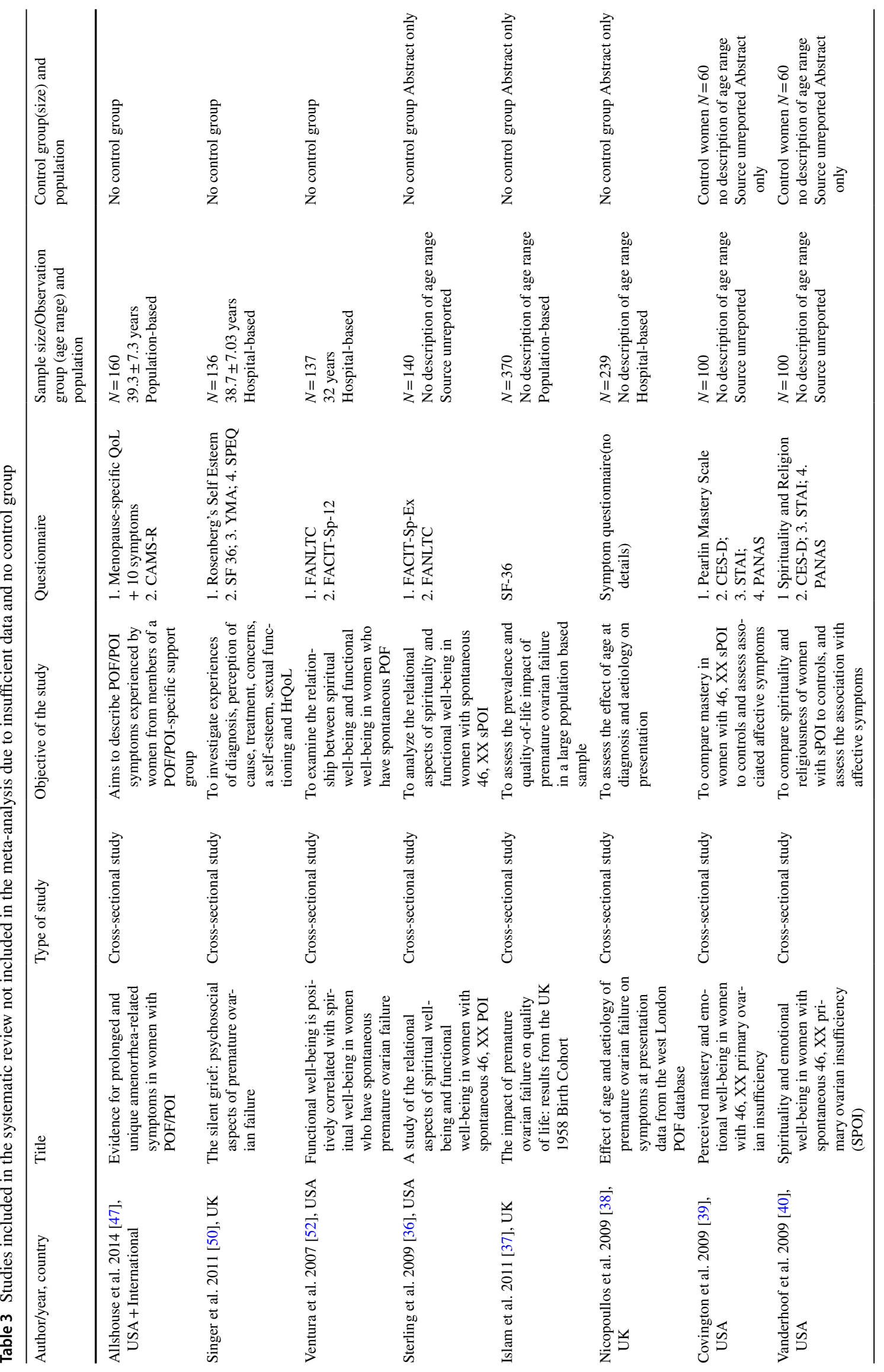




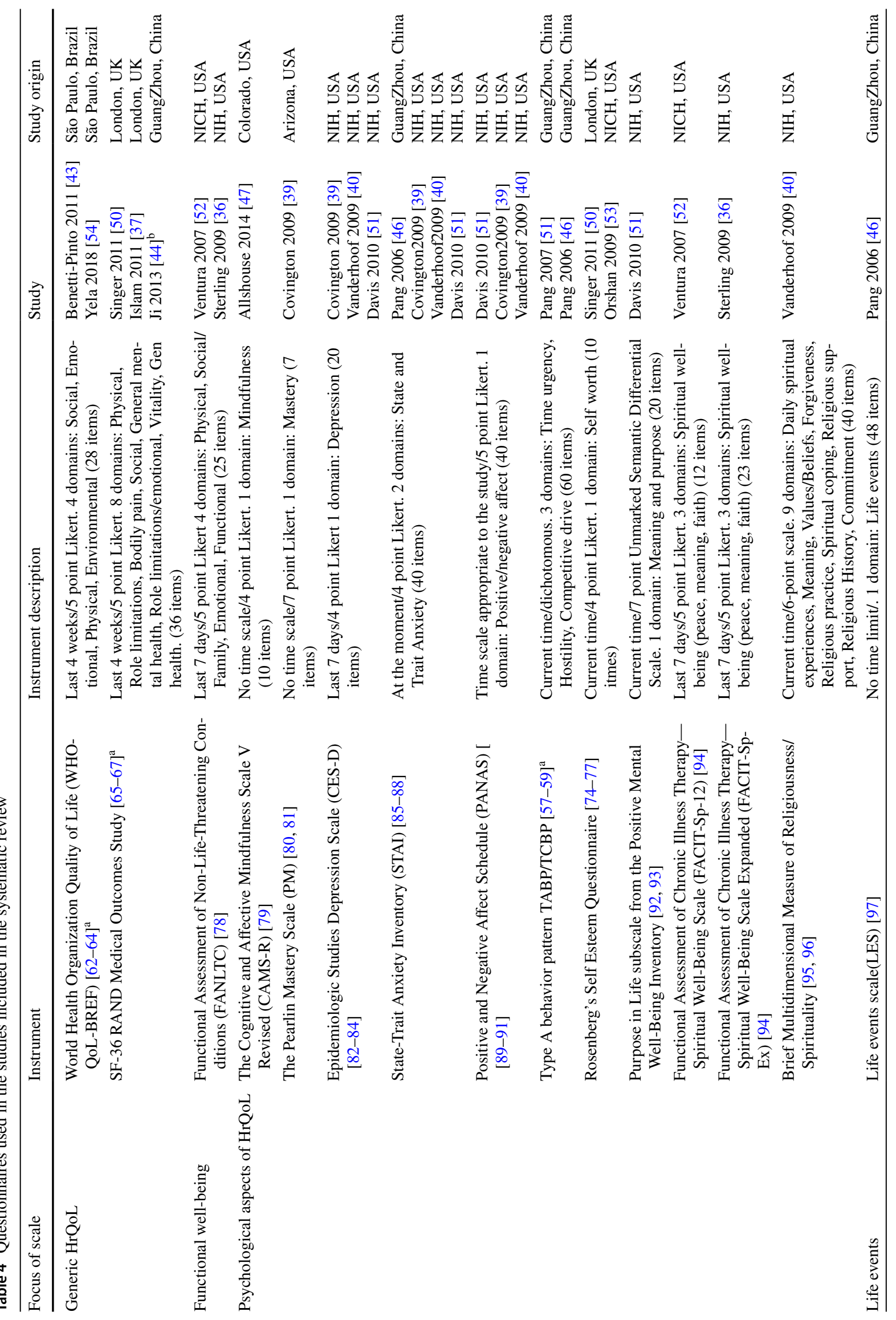




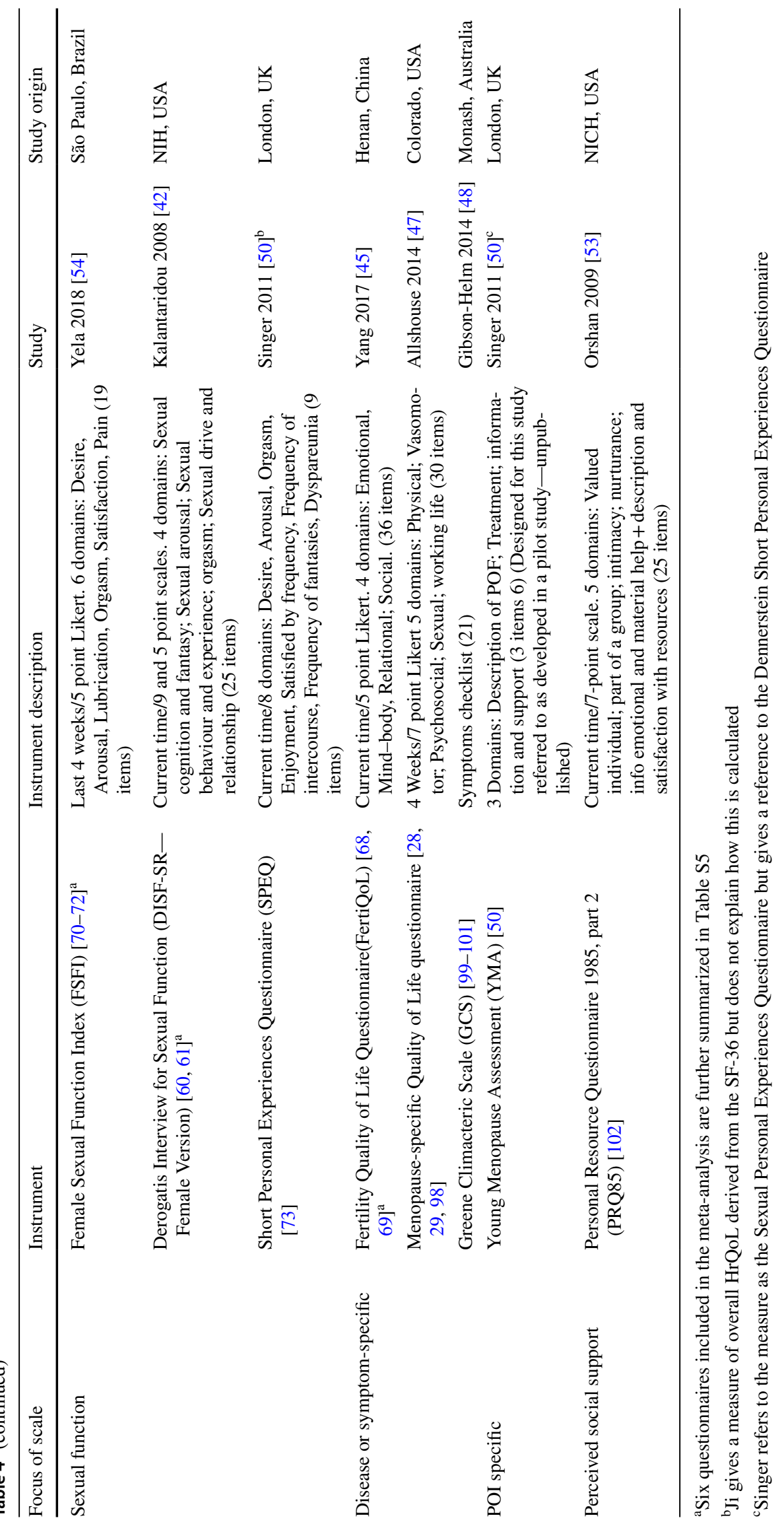




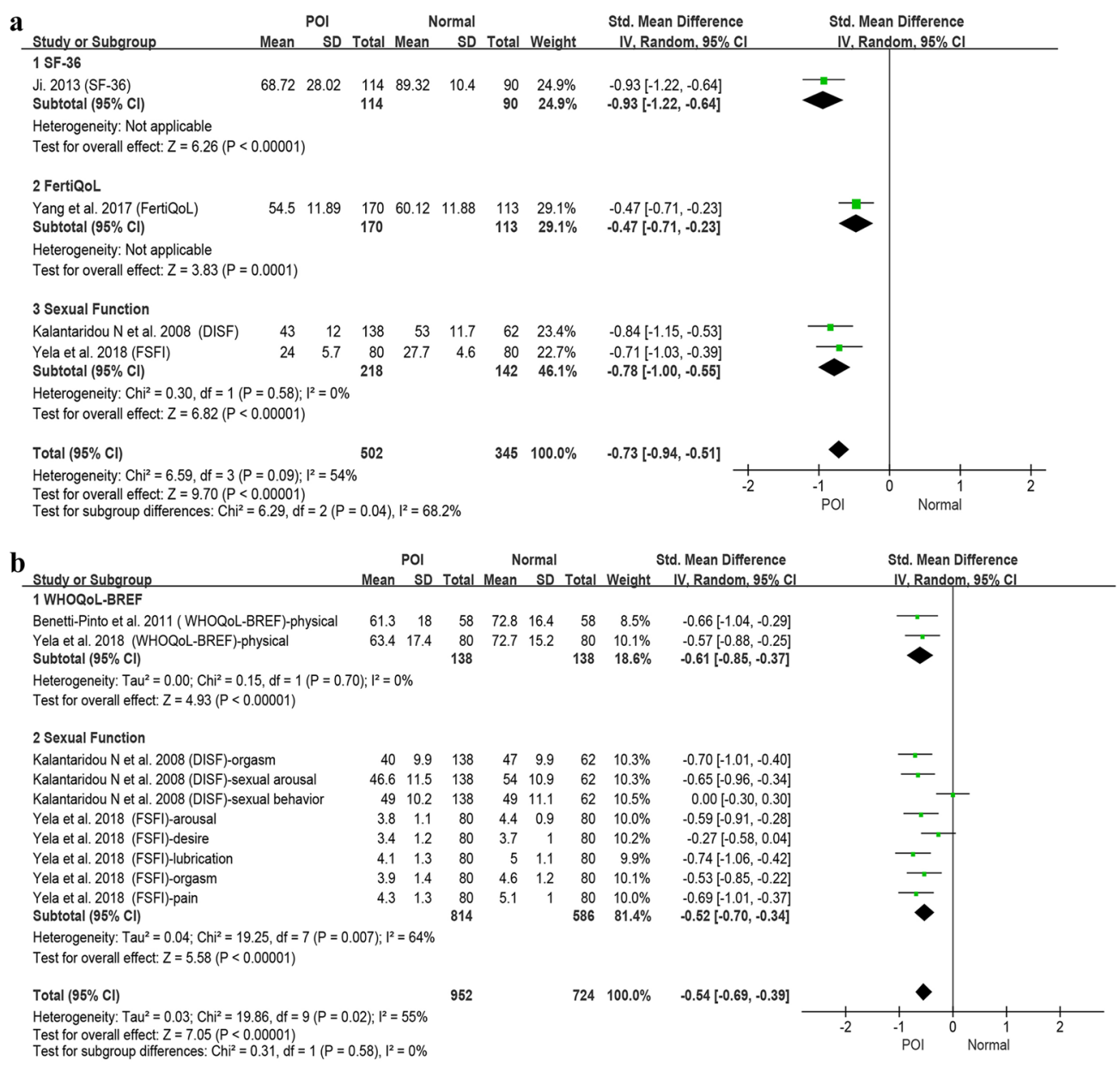

C 1) Higher score refers to better condition

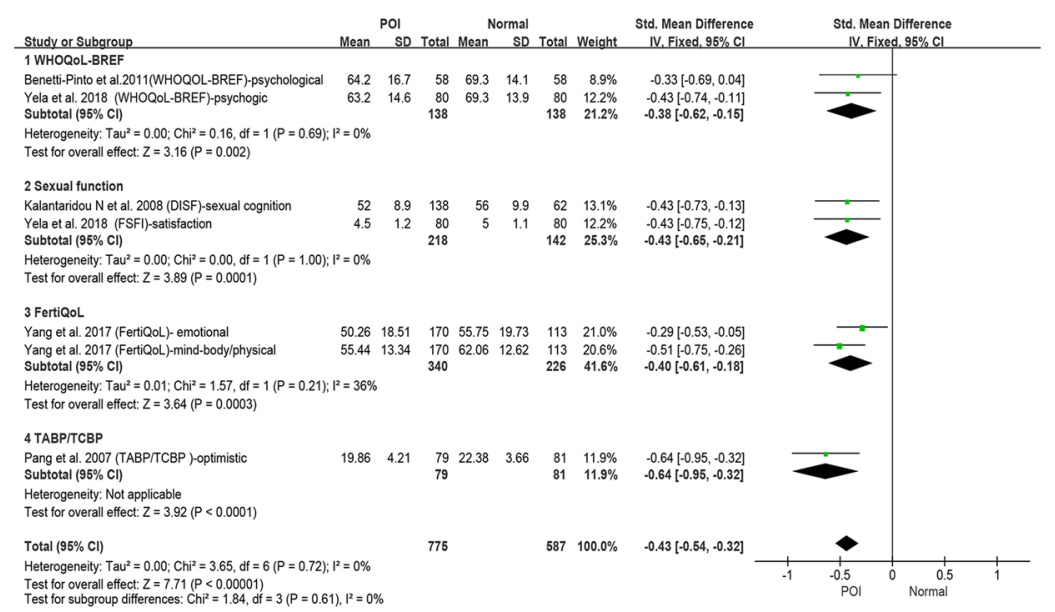

2) Lower score refers to better condition

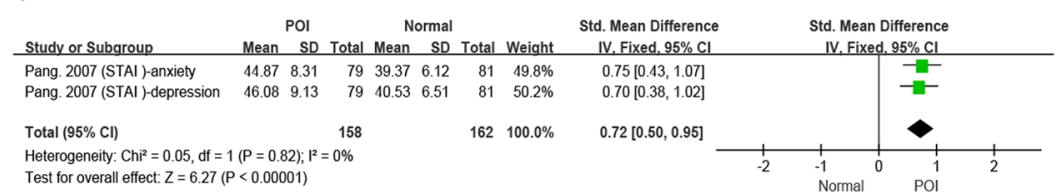

Fig. 2 a Patients with POI compared with normal ovarian reference populations: overall health related quality-of-life (HrQoL). b Patients with POI compared with normal ovarian reference populations: phys- ical functioning. c Patients with POI compared with normal ovarian reference populations: mental health. d Patients with POI compared with normal ovarian reference populations: social functioning 


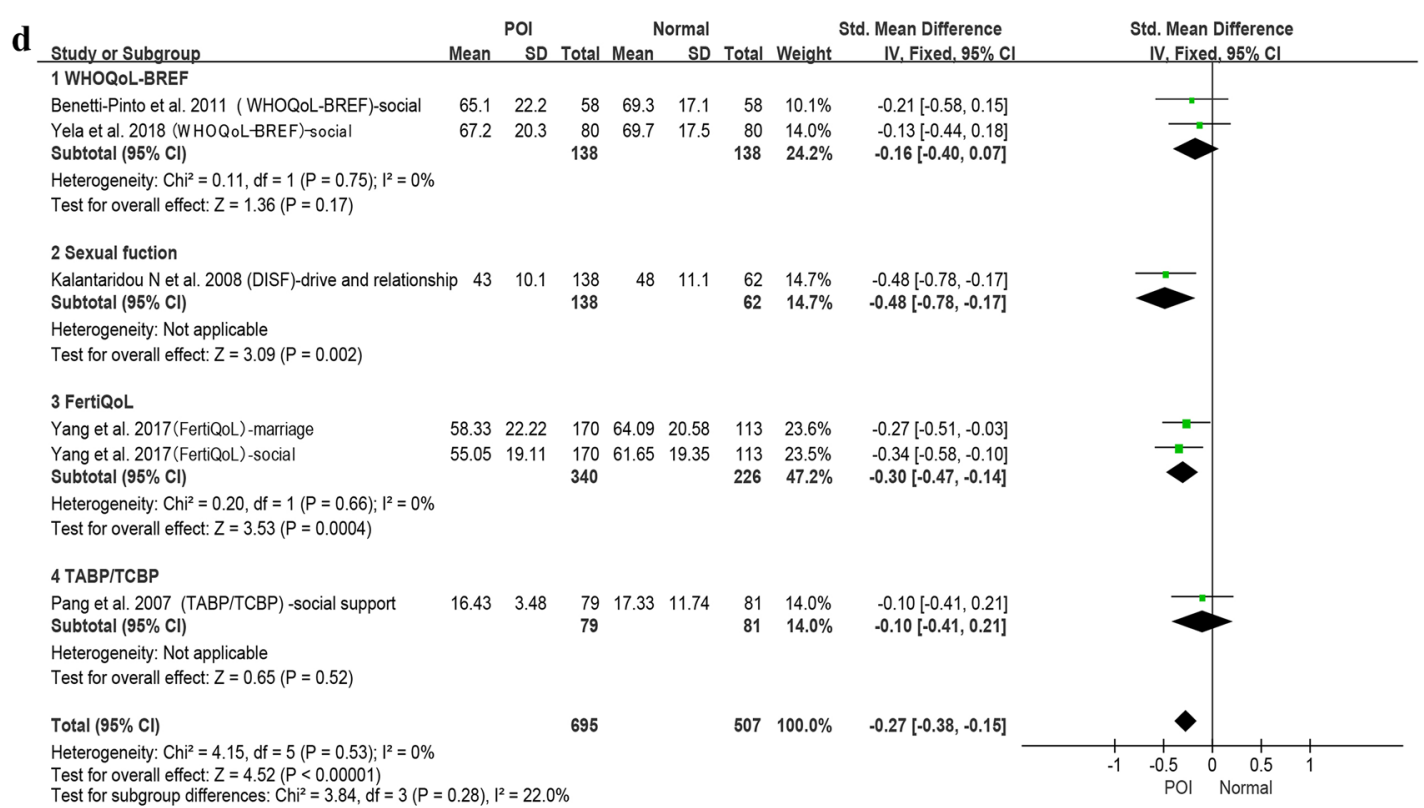

Fig. 2 (continued)

heterogeneity can be considered moderate. To address the heterogeneity, a subgroup analysis ( 2 studies included) was performed to separately examine the measures of sexual functioning (Fig. 2a3) $(\mathrm{SMD}=-0.78,95 \% \mathrm{CI}-1.00$, $-0.55 ; I^{2}=0 \%$ ) the effect size was medium to large and there was no indication of heterogeneity. The largest effect size (large) was found for 'overall HrQoL' as measured by the SF-36 (-0.93, 95\% CI $-1.22,-0.64)$.

In regard to the physical functioning aspects of HrQoL (Fig. 2b), this was measured by four studies using nine different indicators. The results again showed moderate pooled effect size and moderate heterogeneity (pooled $\mathrm{SMD}=-0.54,95 \% \mathrm{CI}-0.69,-0.39 ; I^{2}=55 \%$ ) as compared to a normal ovarian control group. The sexual function (2 studies included) measures explained the heterogeneity where these alone demonstrated substantial heterogeneity $\left(I^{2}=64 \%\right)$ but with a medium effect size (SMD $=0-0.52$, $95 \%$ CI $\left.-0.70,-0.34 ; I^{2}=64 \%\right)$. The largest effect size (moderate) was found for 'Lubrication' as measured by the FSFI $(-0.74,95 \% \mathrm{CI}-1.06,-0.42)$.

In the mental health area (Fig. 2c1, 2), the studies agreed that there was a lower level of mental health in the POI group than was found in the controls however the pooled effect size was small $\left[1 . \mathrm{SMD}=-0.43,95 \% \mathrm{CI}-0.54,-0.32 ; I^{2}=0 \%\right.$ (higher score $=$ better Fig. 2c1); $2 . \mathrm{SMD}=0.72,95 \%$ CI 0.50 , $0.95 ; I^{2}=0 \%$ (lower score = better Fig. 2c2)]. The largest effect size (moderate) was found for 'Optimism' as measured by the TABP/TABC $(-0.64,95 \% \mathrm{CI}-0.95,-0.32)$.

The social functioning domain (Fig. 2d) was addressed by five of the six studies, the pooled effect size was small with no heterogeneity (pooled SMD $=-0.27,95 \% \mathrm{CI}-0.38$, $\left.-0.15 ; I^{2}=0 \%\right)$. The largest effect size (moderate) was found for 'Drive and relationship' in the DISF $(-0.48,95 \%$ CI - 0.78, - 0.17).

Ji [44] has calculated a total QoL score for the SF-36. There is not information on how this was calculated. For discussion on this issue see by Lins and Martins Carvalho (2016) https://doi.org/10.1177/2050312116671725.

\section{Discussion}

Nineteen studies reported the empirical measurement of HrQoL among patients with POI. Reports of the impact of POI on different aspects of HrQoL differed between studies. However, impaired physical, psychological and general health was reported across all areas of HrQoL. There were no articles prior to 2006 and studies used a variety of HrQoL instruments both generic and condition specific although only one measure was specially designed for POI [50]. Although subjective experiences of patients with POI have received more attention from the medical profession in the past decade, relevant and valid evaluation instruments have not been developed, and long-term follow-up studies of HrQoL have not been carried out.

The six controlled studies included in the meta-analysis demonstrated that overall $\mathrm{HrQoL}$ in patients with POI/POF is lower than individuals with normal ovarian functioning with low to medium pooled effect sizes [41-45, 54]. The moderate heterogeneity in the general measure of $\mathrm{HrQoL}$ appears to be due to the different concept being measured under the term HrQoL. It may also come from the different 
socioeconomic groups being included in the various studies. Information on socioeconomic status was sparsely reported and it was not possible for us to make an assessment of the influence of this moderator.

The finding that studies concerning $\mathrm{HrQoL}$ in relation to POI were not found prior to 2006 may be related to fact that the definition of POI had not been standardized. Recent guidelines from the European Society of Human Reproduction and Embryology, published in 2015 [2], coincide with the beginning of investigations into HrQoL in POI. However, some variation in diagnostic criteria is evident. Some studies used broader age intervals, and the levels of Follicle-Stimulating Hormone (FSH), which is a very important indicator of POI diagnosis [2], were vague. This may lead to heterogeneity of the results.

The factors measured in the six studies in the meta-analysis varied and included: fertility, sexual function, anxiety, depression, menopausal symptoms. Although all the measurements were cross-sectional, the concepts measures could all be considered to have long-term effects and would vary according to, for example, diagnostic age, marriage condition or education. In one study [45], an association was investigated between personal character traits and the impact of POI this highlighted the patient's response to the stress of a POI diagnosis and of living with the condition.

Geographical diversity is apparent from our review. It is noted that studies were found in five countries and included one multi-national study [47]. Studies taking a cross-cultural perspective were not conducted. This highlights the possibility of cultural bias in the results [103]. The sparsity of these studies may be due to the lack of a single agreed and validated condition specific instrument translated into multiple language. In addition, despite substantial clinical studies on the use of traditional medicine with this condition, there is a lack of controlled studies that can be used as evidence of treatment effects.

The large number of instruments used (23) in 19 studies with a very low repetition rate, indicates that there is no common view concerning instruments. In some studies, the generic instruments were used to address a comprehensive array of domains of QoL, however, this focus may have limited the sensitivity to detect subtle aspects of POI. It is interesting to speculate on what we did not find, which was the patient perspective. The instrument designed for POI by Singer [50] for their study was based on 'clinical experience' and covered the areas of 'About your POF/young menopause', 'Treatment', and 'Information and Support'. For many patients, there are concerns about the implications of the treatment and of possible long-term side effects which might be more meaningful to the patient [104, 105] and yet these aspects were not investigated. Some studies choose questionnaires that are specific for similar conditions such as menopause or infertility, however, even though the symptoms may be similar, the patients' experiences and requirements may not be the same [47, 48, 54]. It also must be considered that these questionnaires may not be sensitive to all patients with POI. Although the majority of the questionnaires used to measure $\mathrm{HrQoL}$ in these studies had good psychometric properties, none of them had evidence to confirm the sensitivity and specificity of the instruments in relation to POI. There were ten studies [36, 39, 40, 46, 47, 50-54] that used a combination of questionnaires to capture more comprehensive information. However, mood, symptom, and fertility questions specific for women with POI were lacking $[47,50]$.

\section{Strengths and limitations}

Some limitations of the study need to be taken into consideration. It is possible that some studies have been missed due to the use of different terms for POI or in languages that were not included in the databases we examined. There were some studies that were only published as Abstracts and although we tried to contact these researchers we were unable to obtain more information. Our study has the strength of including both European and Asian databases. Those databases that were searched are those that have the highest likelihood of finding studies of HrQoL and POI.

\section{Conclusion and future recommendations}

This literature review and meta-analysis gives new information on HrQoL in patients with POI. In this review, the magnitude of the subjective effects is found to vary with effect sizes between low and medium. The largest effect sizes were found in the area of sexual function and general HrQoL. Cross-cultural approaches and international collaboration were found in only one study. Additional studies are recommended to make a stratified comparison of patients, larger sample sizes to identify real changes in outcomes and longterm follow-ups need to be done in order to have sufficient information for evidence based clinical practice decisions. Future research should focus on developing condition specific and sensitive assessments of the effect of POI based on the patient perspective. This can be achieved through focus groups with the aim of achieving a broader understanding of the outcome domains that are relevant to this population.

Funding This study was funded by National Key R\&D Projects: International Cooperation Research on Evaluation of the Effect of Acupuncture on Superiority Diseases (Grant Number 2017YFC1703600) and National 13th Five-Year Plan: Evaluation of the Effect of Acupuncture on Ovarian Function (Grant Number 2017YFC1703603). 


\section{Compliance with ethical standards}

Ethical approval We will report this review in accordance with the Preferred Reporting Items for Systematic Review and Meta-Analysis statement. A submission to the ethics committee of the Clinical Basic Medicine Institute, China Academy of Chinese Medical Sciences considered that an ethics review was not required (ref 2019/1).

Open Access This article is distributed under the terms of the Creative Commons Attribution 4.0 International License (http://creativeco mmons.org/licenses/by/4.0/), which permits unrestricted use, distribution, and reproduction in any medium, provided you give appropriate credit to the original author(s) and the source, provide a link to the Creative Commons license, and indicate if changes were made.

\section{References}

1. Torrealday, S., Kodaman, P., \& Pal, L. (2017). Premature ovarian insufficiency-An update on recent advances in understanding and management. F1000research, 6, 2069.

2. Webber, L., Davies, M., Anderson, R., Bartlett, J., Braat, D., Cartwright, B., et al. (2016). ESHRE Guideline: Management of women with premature ovarian insufficiency. Human Reproduction, 31(5), 926-927.

3. Zhou, K., Jiang, J., Wu, J., \& Liu, Z. (2013). Electroacupuncture modulates reproductive hormone levels in patients with primary ovarian insufficiency: Results from a prospective observational study. Evidence-Based Complementary and Alternative Medicine, 2013, 1-7. https://doi.org/10.1155/2013/657234.

4. Panay, N., \& Kalu, E. (2009). Management of premature ovarian failure. Best Practice \& Research Clinical Obstetrics \& Gynaecology, 23(1), 129-140.

5. Coulam, C. B., Adamson, S. C., \& Annegers, J. F. (1986). Incidence of premature ovarian failure. Obstetrics and Gynecology, 67(4), 604-606.

6. Lagergren, K., Hammar, M., Nedstrand, E., Bladh, M., \& Sydsjö, G. (2018). The prevalence of primary ovarian insufficiency in Sweden; A national register study. BMC Women's Health, 18(1), 175.

7. Haller-Kikkatalo, K., Uibo, R., Kurg, A., \& Salumets, A. (2015). The prevalence and phenotypic characteristics of spontaneous premature ovarian failure: A general population registry-based study. Human Reproduction, 30(5), 1229-1238. https://doi. org/10.1093/humrep/dev021.

8. Maclaran, K., \& Panay, N. (2015). Current concepts in premature ovarian insufficiency. Womens Health (London), 11(2), 169-182. https://doi.org/10.2217/whe.14.82.

9. Cohen, J., Chabbert-Buffet, N., \& Darai, E. (2015). Diminished ovarian reserve, premature ovarian failure, poor ovarian responder-A plea for universal definitions. Journal of Assisted Reproduction and Genetics, 32(12), 1709-1712. https://doi. org/10.1007/s10815-015-0595-y.

10. Nelson, L. M. (2009). Primary ovarian insufficiency. New England Journal of Medicine, 360(6), 606.

11. Bakalov, V. K., Anasti, J. N., Calis, K. A., Vanderhoof, V. H., Premkumar, A., Chen, S., et al. (2005). Autoimmune oophoritis as a mechanism of follicular dysfunction in women with 46, XX spontaneous premature ovarian failure. Fertility and Sterility, 84(4), 958-965. https://doi.org/10.1016/j.fertnstert.2005.04.060.
12. Podfigurna-Stopa, A., Czyzyk, A., Grymowicz, M., Smolarczyk, R., Katulski, K., Czajkowski, K., et al. (2016). Premature ovarian insufficiency: The context of long-term effects. Journal of Endocrinological Investigation, 39(9), 983-990. https://doi. org/10.1007/s40618-016-0467-z.

13. Luisi, S., Orlandini, C., Biliotti, G., Scolaro, V., De Felice, G., Regini, C., et al. (2013). Hormone replacement therapy in menopause and in premature ovarian insufficiency. Minerva Ginecologica, 65(6), 607-620.

14. Slopien, R. (2018). Mood disorders in women with premature ovarian insufficiency. Przeglad Menopauzalny, 17(3), 124-126. https://doi.org/10.5114/pm.2018.78556.

15. Daan, N. M., Muka, T., Koster, M. P., Roeters van Lennep, J. E., Lambalk, C. B., Laven, J. S., et al. (2016). Cardiovascular risk in women with premature ovarian insufficiency compared to premenopausal women at middle age. Journal of Clinical Endocrinology and Metabolism, 101(9), 3306-3315. https://doi. org/10.1210/jc.2016-1141.

16. Hamoda, H. (2017). The British Menopause Society and Women's Health Concern recommendations on the management of women with premature ovarian insufficiency. Post Reproductive Health, 23(1), 22-35. https://doi.org/10.1177/205336911769935 8.

17. Szeliga, A., Maciejewska-Jeske, M., \& Meczekalski, B. (2018). Bone health and evaluation of bone mineral density in patients with premature ovarian insufficiency. Przeglad Menopauzalny, 17(3), 112-116. https://doi.org/10.5114/pm.2018.78552.

18. Calik-Ksepka, A., Grymowicz, M., Rudnicka, E., Skorska, J., Machura, P., Pieta, W., et al. (2018). Signs and symptoms, evaluation, and management of genitourinary tract consequences of premature ovarian insufficiency. Przeglad Menopauzalny, 17(3), 131-134. https://doi.org/10.5114/pm.2018.78558.

19. Slopien, R. (2018). Neurological health and premature ovarian insufficiency-pathogenesis and clinical management. Przeglad Menopauzalny, 17(3), 120-123. https://doi.org/10.5114/ pm.2018.78555.

20. Lokkegaard, E., Jovanovic, Z., Heitmann, B. L., Keiding, N., Ottesen, B., \& Pedersen, A. T. (2006). The association between early menopause and risk of ischaemic heart disease: Influence of hormone therapy. Maturitas, 53(2), 226-233. https://doi. org/10.1016/j.maturitas.2005.04.009.

21. The Whoqol Group. (1998). The World Health Organization Quality of Life Assessment (WHOQOL): Development and general psychometric properties. Social Science and Medicine, 46(12), 1569-1585.

22. Garner, B. R., Scott, C. K., Dennis, M. L., \& Funk, R. R. (2014). The relationship between recovery and health-related quality of life. Journal of Substance Abuse Treatment, 47(4), 293-298. https://doi.org/10.1016/j.jsat.2014.05.006.

23. Karimi, M., \& Brazier, J. (2016). Health, health-related quality of life, and quality of life: What is the difference? Pharmacoeconomics, 34(7), 645-649. https://doi.org/10.1007/s4027 3-016-0389-9.

24. Dobhal, M., Juneja, M., Jain, R., Sairam, S., \& Thiagarajan, D. (2014). Health-related quality of life in children with cerebral palsy and their families. Indian Pediatrics, 51(5), 385-387.

25. Cella, D. F., \& Tulsky, D. S. (1990). Measuring quality of life today: Methodological aspects. Oncology (Williston Park), 4(5), 29-38; discussion 69

26. Longo, E., Badia, M., Begona Orgaz, M., \& Gomez-Vela, M. (2017). Comparing parent and child reports of health-related quality of life and their relationship with leisure participation in children and adolescents with Cerebral Palsy. Research in Developmental Disabilities, 71, 214-222. https://doi.org/10.1016/j. ridd.2017.09.020. 
27. Liberati, A., Altman, D. G., Tetzlaff, J., Mulrow, C., Gotzsche, P. C., Ioannidis, J. P., et al. (2009). The PRISMA statement for reporting systematic reviews and meta-analyses of studies that evaluate healthcare interventions: Explanation and elaboration. BMJ, 339, b2700. https://doi.org/10.1136/bmj.b2700.

28. Hilditch, J. R., Lewis, J., Peter, A., van Maris, B., Ross, A., Franssen, E., et al. (1996). A menopause-specific quality of life questionnaire: Development and psychometric properties. Maturitas, 24(3), 161-175.

29. Kulasingam, S., Moineddin, R., Lewis, J. E., \& Tierney, M. C. (2008). The validity of the Menopause Specific Quality of Life Questionnaire in older women. Maturitas, 60(3), 239-243.

30. Wells, G. A., Shea, B., O'Connell, D., Peterson, J., Welch, V., Losos, M., \& Tugwell, P. (2019). The Newcastle-Ottawa Scale (NOS) for assessing the quality of nonrandomised studies in meta-analyses. Accessed April 17, 2019, from http://www.ohri. ca/programs/clinical_epidemiology/oxford.asp.

31. Aziz, O., Constantinides, V., Tekkis, P. P., Athanasiou, T., Purkayastha, S., Paraskeva, P., et al. (2006). Laparoscopic versus open surgery for rectal cancer: A meta-analysis. Annals of Surgical Oncology, 13(3), 413-424.

32. Peter, M., \& Fayers, D. M. (2007). Quality of Life: The assessment, analysis and interpretation of patient-reported outcomes. Chichester: Wiley.

33. Fu, R., Vandermeer, B. W., Shamliyan, T. A., O’Neil, M. E., Yazdi, F., Fox, S. H., et al. (2008). Handling continuous outcomes in quantitative synthesis. In Methods guide for comparative effectiveness reviews. AHRQ Publication No. 13-EHC103EF. Rockville, MD: Agency for Healthcare Research and Quality.

34. Cohen, J. (1988). Statistical power analysis for the behavioral sciences. Hillsdale, NJ: Lawrence Erlbaum Associates.

35. Higgins, J. P., Thompson, S. G., Deeks, J. J., \& Altman, D. G. (2003). Measuring inconsistency in meta-analyses. BMJ, 327(7414), 557-560. https://doi.org/10.1136/bmj.327.7414.557.

36. Sterling, E. W., Fitzgerald, O. R., Vanderhoof, V. H., Covington, S. N., Koziol, D. E., \& Nelson, L. M. (2009). A study of the relational aspects of spiritual well-being and functional well-being in women with spontaneous 46 , XX primary ovarian insufficiency. Fertility and Sterility, 92(3), S130-S130.

37. Islam, R., \& Cartwright, R. (2011). The impact of premature ovarian failure on quality of life: Results from the UK 1958 Birth Cohort [Abstract]. In Abstracts of the 27th annual meeting of ESHRE, Stockholm, Sweden, 3-6 July 2011.

38. Nicopoullos, J., Grech, H., Domoney, C., Horner, E., Kalu, E., Bellone, C., et al. (2009). Effect of age and aetiology of premature ovarian failure on symptoms at presentation data from the west London POF database. Maturitas, 63(63), S29-S30.

39. Covington, S. N., Davis, M. C., Vanderhoof, V. H., Koziol, D. E., \& Nelson, L. M. (2009). Perceived mastery and emotional well-being in women with $46, \mathrm{XX}$ primary ovarian insufficiency. Fertility and Sterility, 92(3), S33-S33.

40. Vanderhoof, V. H., Davis, M., Pollack, J. M., Covington, S. N., Koziol, D. E., \& Nelson, L. M. (2009). Spirituality and emotional well-being in women with spontaneous $46, \mathrm{XX}$ primary ovarian insufficiency (SPOI). Fertility and Sterility, 92(3), S133-S133.

41. Pang Zhenmiao, L. J., \& Gaopei, D. (2007). Investigations of personality characteristics and mental health status in patients with premature ovarian failure. Journal of Clinical Psychosomatic Diseases, 13(5), 428-430.

42. Kalantaridou, S. N., Vanderhoof, V. H., Calis, K. A., Corrigan, E. C., Troendle, J. F., \& Nelson, L. M. (2008). Sexual function in young women with spontaneous $46, \mathrm{XX}$ primary ovarian insufficiency. Fertility and Sterility, 90(5), 1805-1811. https:// doi.org/10.1016/j.fertnstert.2007.08.040.
43. Benetti-Pinto, C. L., de Almeida, D. M., \& Makuch, M. Y. (2011). Quality of life in women with premature ovarian failure. Gynecological Endocrinology, 27(9), 645-649.

44. Ji, X. (2013). Clinical study on the relationship between syndrome types differentiation of TCM and quality of life in premature ovarian failure. Chengdu: Chengdu University of TCM, CNKI.

45. Yang Li, Z. F., \& Yuezhi, D. (2017). Study on quality of fertility in patients with premature ovarian failure. Chinese Nursing Research, 31(1), 115-117.

46. Pang, Z. (2006). The demonstration study of the relationship between the social/psychology factors in patients with POF. Guang Zhou: Guang Zhou University of Traditional Chinese Medicine.

47. Allshouse, A. A., Semple, A. L., \& Santoro, N. F. (2015). Evidence for prolonged and unique amenorrhea-related symptoms in women with premature ovarian failure/primary ovarian insufficiency. Menopause: The Journal of the North American Menopause Society, 22(2), 166-174.

48. Gibson-Helm, M., Teede, H., \& Vincent, A. (2014). Symptoms, health behavior and understanding of menopause therapy in women with premature menopause. Climacteric: The Journal of the International Menopause Society, 17(6), 8.

49. Schmidt, P. J., Luff, J. A., Haq, N. A., Vanderhoof, V. H., Koziol, D. E., Calis, K. A., et al. (2011). Depression in women with spontaneous 46, XX primary ovarian insufficiency. Journal of Clinical Endocrinology and Metabolism, 96(2), 278-287.

50. Singer, D., Mann, E., Hunter, M. S., Pitkin, J., \& Panay, N. (2011). The silent grief: Psychosocial aspects of premature ovarian failure. Climacteric: The Journal of the International Menopause Society, 14(4), 428-437.

51. Davis, M., Ventura, J. L., Wieners, M., Covington, S. N., Vanderhoof, V. H., Ryan, M. E., et al. (2010). The psychosocial transition associated with spontaneous $46, \mathrm{XX}$ primary ovarian insufficiency: Illness uncertainty, stigma, goal flexibility, and purpose in life as factors in emotional health. Fertility and Sterility, 93(7), 2321-2329. https://doi.org/10.1016/j.fertnstert.2008.12.122.

52. Ventura, J. L., Fitzgerald, O. R., Koziol, D. E., Covington, S. N., Vanderhoof, V. H., Calis, K. A., et al. (2007). Functional well-being is positively correlated with spiritual well-being in women who have spontaneous premature ovarian failure. Fertility and Sterility, 87(3), 584-590. https://doi.org/10.1016/j.fertn stert.2006.07.1523.

53. Orshan, S. A., Ventura, J. L., Covington, S. N., Vanderhoof, V. H., Troendle, J. F., \& Nelson, L. M. (2009). Women with spontaneous 46, XX primary ovarian insufficiency (hypergonadotropic hypogonadism) have lower perceived social support than control women. Fertility and Sterility, 92(2), 688-693.

54. Yela, D. A., Soares, P. M., \& Benetti-Pinto, C. L. (2018). Influence of sexual function on the social relations and quality of life of women with premature ovarian insufficiency. Revista Brasileira de Ginecologia e Obstetricia, 40(2), 66-71. https://doi. org/10.1055/s-0037-1615289.

55. Kang, H., Zhao, Y., Li, C., Chen, Y., Tang, K., Yang, L., et al. (2015). Integrating clinical indexes into four-diagnostic information contributes to the Traditional Chinese Medicine (TCM) syndrome diagnosis of chronic hepatitis B. Scientific Reports, 5, 9395. https://doi.org/10.1038/srep09395.

56. Bertels, J., Demoulin, C., Franco, A., \& Destrebecqz, A. (2013). Side effects of being blue: Influence of sad mood on visual statistical learning. PLOS ONE, 8(3), e59832. https://doi.org/10.1371/ journal.pone.0059832.

57. Zhang, B. Y. (1985). The National Collaborative Study Group for TABP \& CHD: Psychophysiological reaction in cardiovascular disease. Acta Psychologica Sinica, 3, 314-321. 
58. Zhang, S. J., Zhou, Q., Zhang, R. L., Shen, M. X., Wang, X. C., Xie, X. B., et al. (2005). Studies on relations between a type behavior and the infection of choleithiasis. Chinese Journal of Behavioral Medical Science, 14(3), 217-219.

59. Shi, H., Yang, X., Wang, J., Xi, H., Huang, C., He, J., et al. (2013). Type A personality, hostility, time urgency and unintentional injuries among Chinese undergraduates: A matched case-control study. BMC Public Health, 13, 1066. https://doi. org/10.1186/1471-2458-13-1066.

60. Derogatis, L. R. (1997). The Derogatis Interview for Sexual Functioning (DISF/DISF-SR): An introductory report. Journal of Sex and Marital Therapy, 23(4), 291-304. https://doi. org/10.1080/00926239708403933.

61. Meston, C. M., \& Derogatis, L. R. (2002). Validated instruments for assessing female sexual function. Journal of Sex and Marital Therapy, 28(Suppl 1), 155-164. https://doi.org/10.1080/00926 230252851276.

62. Cheung, Y. B., Yeo, K. K., Chong, K. J., Khoo, E. Y., \& Wee, H. L. (2017). Reliability and validity of the English-, Chineseand Malay-Language versions of the World Health Organization Quality of Life (WHOQOL-BREF) Questionnaire in Singapore. Annals of the Academy of Medicine, Singapore, 46(12), 461-469.

63. World Health Organization. (1996). WHOQOL-BREF: Introduction, administration, scoring and generic version of the assessment: Field trial version. http://www.who.int/iris/handle/10665 163529.

64. Vilar, M., Sousa, L. B., \& Simoes, M. R. (2016). The European Portuguese WHOQOL-OLD module and the new facet family/family life: Reliability and validity studies. Quality of Life Research, 25(9), 2367-2372. https://doi.org/10.1007/s1113 6-016-1275-9.

65. Ware, J. E., \& Gandek, B. (1998). Overview of the SF-36 Health Survey and the International Quality of Life Assessment (IQOLA) Project. Journal of Clinical Epidemiology, 51(11), 903-912.

66. Salazar, F. R., \& Bernabe, E. (2015). The Spanish SF-36 in Peru: Factor structure, construct validity, and internal consistency. Asia-Pacific Journal of Public Health, 27(2), 2372-2380. https ://doi.org/10.1177/1010539511432879.

67. Tarlov, A. R., Ware, J. E., Jr., Greenfield, S., Nelson, E. C., Perrin, E., \& Zubkoff, M. (1989). The Medical Outcomes Study: An application of methods for monitoring the results of medical care. JAMA, 262(7), 925-930.

68. Boivin, J., Takefman, J., \& Braverman, A. (2011). The fertility quality of life (FertiQoL) tool: Development and general psychometric properties. Human Reproduction, 26(8), 2084-2091. https ://doi.org/10.1093/humrep/der171.

69. Gao, M., Ji, X., Zhou, L., \& Zhang, Z. (2016). The fertility quality of life (FertiQol) in Chinese infertile women. Translational Andrology \& Urology, 5(Suppl 1), AB084.

70. Crisp, C. C., Fellner, A. N., \& Pauls, R. N. (2015). Validation of the Female Sexual Function Index (FSFI) for web-based administration. International Urogynecology Journal, 26(2), 219-222. https://doi.org/10.1007/s00192-014-2461-3.

71. Stephenson, K. R., Toorabally, N., Lyons, L., \& Meston C, M. (2016). Further validation of the female sexual function index: Specificity and associations with clinical interview data. Journal of Sex and Marital Therapy, 42(5), 448-461. https://doi. org/10.1080/0092623x.2015.1061078.

72. Rosen, R., Brown, C., Heiman, J., Leiblum, S., Meston, C., Shabsigh, R., et al. (2000). The Female Sexual Function Index (FSFI): A multidimensional self-report instrument for the assessment of female sexual function. Journal of Sex and Marital Therapy, 26(2), 191-208. https://doi.org/10.1080/009262300278597.
73. Dennerstein, L., Andersonhunt, M., \& Dudley, E. (2002). Evaluation of a short scale to assess female sexual functioning. Journal of Sex and Marital Therapy, 28(5), 389-397.

74. Rosenberg, M. (1965). Society and the adolescent self-image (Vol. 3(2), pp. 1780-1790). Princeton: Princeton University Press.

75. Alessandri, G., Vecchione, M., Eisenberg, N., \& Laguna, M. (2015). On the factor structure of the Rosenberg (1965) General Self-Esteem Scale. Psychological Assessment, 27(2), 621-635. https://doi.org/10.1037/pas0000073.

76. McMullen, T., \& Resnick, B. (2013). Self-esteem among nursing assistants: Reliability and validity of the Rosenberg Self-Esteem Scale. Journal of Nursing Measurement, 21(2), 335-344.

77. Piyavhatkul, N., Aroonpongpaisal, S., Patjanasoontorn, N., Rongbutsri, S., Maneeganondh, S., \& Pimpanit, W. (2011). Validity and reliability of the Rosenberg Self-Esteem Scale-Thai version as compared to the Self-Esteem Visual Analog Scale. Journal of the Medical Association of Thailand, 94(7), 857-862.

78. Cella, D., \& Nowinski, C. J. (2002). Measuring quality of life in chronic illness: The functional assessment of chronic illness therapy measurement system. Archives of Physical Medicine and Rehabilitation, 83(12 Suppl 2), S10-17. https://doi.org/10.1053/ apmr.2002.36959.

79. Feldman, G., Hayes, A., Kumar, S., Greeson, J., \& Laurenceau, J. P. (2007). Mindfulness and emotion regulation: The development and initial validation of the Cognitive and Affective Mindfulness Scale-Revised (CAMS-R). Journal of Psychopathology \& Behavioral Assessment, 29(3), 177.

80. Gordon, J. R., Malcarne, V. L., Roesch, S. C., Roetzheim, R. G., \& Wells, K. J. (2018). Structural validity and measurement invariance of the Pearlin Mastery Scale in Spanish-speaking primary care patients. Evaluation and the Health Professions, 41(3), 393-399. https://doi.org/10.1177/0163278718774942.

81. Pearlin, L. I., \& Schooler, C. (1978). The structure of coping. Journal of Health and Social Behavior, 19(1), 2-21.

82. Siddaway, A. P., Wood, A. M., \& Taylor, P. J. (2017). The Center for Epidemiologic Studies-Depression (CES-D) scale measures a continuum from well-being to depression: Testing two key predictions of positive clinical psychology. Journal of Affective Disorders, 213, 180-186. https://doi.org/10.1016/j.jad.2017.02.015.

83. Ls, R. (1977). The CES-D scale: A self-report depression scale for research in the general population. Applied Psychological Measurement, 1, 385-401.

84. Schroevers, M. J., Sanderman, R., van Sonderen, E., \& Ranchor, A. V. (2000). The evaluation of the Center for Epidemiologic Studies Depression (CES-D) scale: Depressed and positive affect in cancer patients and healthy reference subjects. Quality of Life Research, 9(9), 1015-1029.

85. Guillen-Riquelme, A., \& Buela-Casal, G. (2014). Meta-analysis of group comparison and meta-analysis of reliability generalization of the State-Trait Anxiety Inventory Questionnaire (STAI). Revista Española de Salud Pública, 88(1), 101-112. https://doi. org/10.4321/s1135-57272014000100007.

86. Marteau, T. M., \& Bekker, H. (1992). The development of a sixitem short-form of the state scale of the Spielberger State-Trait Anxiety Inventory (STAI). British Journal of Clinical Psychology, 31(Pt 3), 301-306.

87. Roberts, K. E., Hart, T. A., \& Eastwood, J. D. (2016). Factor structure and validity of the State-Trait Inventory for Cognitive and Somatic Anxiety. Psychological Assessment, 28(2), 134-146. https://doi.org/10.1037/pas0000155.

88. Shek, D. T. L. (1988). Reliability and factorial structure of the Chinese version of the State-Trait Anxiety Inventory. Journal of Psychopathology \& Behavioral Assessment, 10(4), 303-317.

89. Ostir, G. V., Smith, P. M., Smith, D., \& Ottenbacher, K. J. (2005). Reliability of the positive and negative affect schedule (PANAS) 
in medical rehabilitation. Clinical Rehabilitation, 19(7), 767769. https://doi.org/10.1191/0269215505cr894oa.

90. Watson, D., Clark, L. A., \& Tellegen, A. (1988). Development and validation of brief measures of positive and negative affect: The PANAS scales. Journal of Personality and Social Psychology, 54(6), 1063-1070.

91. Brdar, I. (2014). Positive and Negative Affect Schedule (PANAS). In A. C. Michalos (Ed.), Encyclopedia of quality of life and well-being research (p. 1511). Dordrecht: Springer.

92. Crumbaugh, J. C. (1968). Cross-validation of purpose-in-life test based on Frankl's concepts. Journal of Individual Psychology, 24(1), 74-81.

93. Chang, R. H., \& Dodder, R. A. (1983). The modified purpose in life scale: A cross-national validity study. International Journal of Aging and Human Development, 18(3), 207-217.

94. Brady, M. J., Peterman, A. H., Fitchett, G., Mo, M., \& Cella, D. (1999). A case for including spirituality in quality of life measurement in oncology. Psycho-Oncology, 8, 417-428.

95. John E. Fetzer Institute. (1999). Multidimensional measurement of religiousness/spiritualtiy for use in health research. Kalamazoo, MI: National Institute on Aging/Fetzer Institute.

96. Traphagan, J. W. (2015). Multidimensional measurement of religiousness/spirituality for use in health research in cross-cultural perspective. Research on Aging, 27(4), 387-419.

97. Dourado, C. S., Souza, C. B., Castro, D. S., Zandonade, E., Miotto, M., \& Amorim, M. H. C. (2018). Association between life events after diagnosis of breast cancer and metastasis. Ciência \& Saúde Coletiva, 23(2), 471-480. https://doi. org/10.1590/1413-81232018232.15672015.

98. Sydora, B. C., Fast, H., Campbell, S., Yuksel, N., Lewis, J. E., \& Ross, S. (2016). Use of the Menopause-Specific Quality of Life (MENQOL) questionnaire in research and clinical practice: A comprehensive scoping review. Menopause, 23(9), 1038-1051. https://doi.org/10.1097/gme.0000000000000636.
99. Travers, C., O’Neill, S. M., King, R., Battistutta, D., \& Khoo, S. K. (2005). Greene Climacteric Scale: Norms in an Australian population in relation to age and menopausal status. Climacteric, 8(1), 56-62. https://doi.org/10.1080/13697130400013443.

100. Vasconcelos-Raposo, J., Coelho, E., Fernandes, H. M., Rodrigues, C., Moreira, H., \& Teixeira, C. (2012). Factor structure and normative data of the Greene Climacteric Scale among postmenopausal Portuguese women. Maturitas, 72(3), 256-262. https ://doi.org/10.1016/j.maturitas.2012.04.003.

101. Chen, R. Q., Davis, S. R., Wong, C. M., \& Lam, T. H. (2010). Validity and cultural equivalence of the standard Greene Climacteric Scale in Hong Kong. Menopause, 17(3), 630-635. https:// doi.org/10.1097/gme.0b013e3181ca0adb.

102. Brandt, P. A., \& Weinert, C. (1981). The PRQ-A social support measure. Nursing Research, 30(5), 277-280.

103. Bullinger, M. (1997). The challenge of cross-cultural quality of life assessment. Psychology \& Health, 12(6), 815-825.

104. Waters, E., Davis, E., Ronen, G. M., Rosenbaum, P., Livingston, M., \& Saigal, S. (2009). Quality of life instruments for children and adolescents with neurodisabilities: How to choose the appropriate instrument. Developmental Medicine and Child Neurology, 51(8), 660-669. https://doi.org/10.1111/j.1469-8749.2009.03324 .x.

105. Wiebe, S., Guyatt, G., Weaver, B., Matijevic, S., \& Sidwell, C. (2003). Comparative responsiveness of generic and specific quality-of-life instruments. Journal of Clinical Epidemiology, $56(1), 52-60$.

Publisher's Note Springer Nature remains neutral with regard to jurisdictional claims in published maps and institutional affiliations. 